\title{
EL MACROGÉNERO ESCENARIOS: UNA EXPERIENCA DE \\ ENZEÑANZA A LO LARGO DEL CURRICULUM DESDE LA \\ LINGÜÍSTICA SISTÉMICO-FUNCIONAL
}

\section{THE MACROGENRE SCENARIO: AN EXPERIENCE OF TEACHING \\ ACROSS THE CURRICULUM FROM SYSTEMIC FUNCTIONAL \\ LINGUISTICS}

\author{
Yanina Elisabeth García (UFLO) \\ isp.garciay@gmail.com \\ Estela Inés Moyano (UNGS/UNaB/UFLO) \\ estelaimoyano@gmail.com
}

\begin{abstract}
RESUMEN: Este artículo aborda la educación lingüística, desde una práctica a lo largo del currículum, a través de una propuesta didáctica explícita enfocada en los géneros disciplinares basada en la lingüística sistémico-funcional. Toma la experiencia realizada con estudiantes de dos asignaturas de la carrera de Administración de Empresas, en la Universidad de Flores en Argentina. Para eso, se analiza el macrogénero "Escenarios", atendiendo a su estructura y recursos lingüísticos más significativos en el estrato semánticodiscursivo. Luego, se presentan los fundamentos de la propuesta didáctica que se aplicó a la experiencia y se describen las herramientas de análisis de los textos producidos por los estudiantes en la experiencia a fin de dar cuenta de los resultados obtenidos mediante datos cuantitativos. Por último, se argumenta a favor de una enseñanza explícita de los géneros como un modo de contribuir a la inclusión de estudiantes en la educación superior, y en la comunidad disciplinar y profesional.
\end{abstract}

PALABRAS CLAVES: educación superior; lingüística sistémico-funcional; macrogénero; enseñanza a través del currículum.

\begin{abstract}
This paper approaches educational linguistics, focusing the explicit teaching of reading and writing across the curriculum, following a genre-based theory based on systemic functional linguistics. It reports the experience with students of two courses at the degree of Administration, at Universitiy of Flores in Argentina. To do this, the macrogenre "Scenario" is described, paying attention to its schematic structure and the most significant discoursesemantic resources. Then, the foundations of the pedagogic proposal implemented are presented as well as the analysis tools applied to the texts produced by the students in order to present the quantitative results obtained. Finally, we argue that this explicit genre-based
\end{abstract}


teaching of literacy contributes to the inclusion of students in higher education, and also into the disciplinary and professional community.

KEYWORDS: higher education; systemic functional linguistics; macrogenre; teaching across the curriculum.

\section{Introducción}

Es un consenso extendido, tanto en América Latina como en el mundo, que ingresar y participar en la universidad requiere el aprendizaje de las prácticas letradas propias de la comunidad académica y disciplinar. Sumado a eso, la inclusión de sectores no tradicionales en la educación superior puso en evidencia la necesidad, por parte de las universidades, de atender a una distribución desigual del capital cultural (EZCURRA, 2011a, 2011b; NAVARRO, 2016; ROSE; MARTIN, 2012). Para eso, algunas universidades han desarrollado propuestas que persiguen el objetivo de ofrecer a los y las estudiantes distintas herramientas para desempeñarse en el ámbito académico. Podemos identificar, a grandes rasgos, dos grupos: las propuestas de pre-grado, con talleres iniciales donde se reflexionan y ejercitan las prácticas letradas académicas, y las propuestas que se insertan en el currículum de las disciplinas, reflexionando en torno a los géneros académicos a lo largo de las carreras (MOYANO, 2004).

Dentro de este segundo grupo, la Universidad de Flores en Argentina implementa, desde el 2013, el Programa de Lectura y Escritura Académica (PROLEA), cuyo objetivo es ofrecer a los y las estudiantes herramientas para desarrollar sus habilidades lectoras y las competencias en la escritura (MOYANO, 2018). El modelo supone una asociación entre el docente disciplinar y el docente del programa especializado en el lenguaje, y se enmarca en una propuesta pedagógica que adapta la llevada a cabo por la Escuela de Sídney en el marco de la linguística sistémico funcional (MOYANO, 2007). Desde 2018, la propuesta se implementa en la Facultad de Ciencias Organizacionales y de la Empresa, en las carreras de grado de Licenciatura en Administración y Contador Público.

Para este trabajo, nos remitiremos a una experiencia realizada en el marco de la carrera de Licenciatura en Administración, que busca formar a sus estudiantes para evaluar los distintos aspectos de la organización empresarial: el técnico-económico y el sociohumanístico. Su formación les permitirá adaptar sus conocimientos, habilidades y competencias técnicas y gerenciales para ejercer sus funciones como Administradores, tanto 
en las pequeñas, medianas y grandes empresas como en el ejercicio como profesionales o emprendedores independientes. Específicamente, nos centraremos en el trabajo realizado en torno a la elaboración del macrogénero que dio en llamarse Escenarios, en la asignatura "Organización de empresas", durante el primer cuatrimestre del 2019, y en "Simulación de empresas", en el segundo cuatrimestre del 2018.

En relación a la primera, corresponde al segundo año de la carrera, y comprende a la organización como un sistema complejo de relaciones de autoridad y regulación, así como de información para la decisión y el control. A partir de esa visión, busca que los estudiantes estén en condiciones de realizar un diseño organizativo que permita lograr los objetivos de la empresa, atender a las demandas del contexto y utilizar los recursos humanos y materiales involucrados. En cuanto a la enseñanza del macrogénero Escenarios, se ofrece como una metodología para identificar los problemas complejos que atraviesan el presente, para pensar estratégicamente y tomar decisiones frente a los hechos que se describen en los futuros alternativos. Por último, es importante destacar que los estudiantes habían tenido la experiencia del Programa en una asignatura del primer año.

Por otra parte, la asignatura "Simulación de empresas" corresponde al cuarto y último año de la carrera, y tiene por objetivo la revisión de todas las materias cursadas en el transcurso de la carrera, pero con una aplicación práctica, orientada al funcionamiento cotidiano de una empresa. En este caso, la enseñanza del macrogénero Escenarios está orientada a que los estudiantes puedan desagregar cada contexto con una mayor profundidad y diseñar múltiples futuros para poder ponderar proyectos, organizaciones y su propio ejercicio profesional tomando decisiones. Los estudiantes, además, ya habían participado de la propuesta que el PROLEA ofrece en materias de años anteriores, elaborando otros géneros académicos.

En primer lugar, entonces, caracterizaremos este macrogénero desde la perspectiva de la lingüística sistémico-funcional (MARTIN; ROSE, 2007, 2008; MARTIN, 1994; EGGINS; MARTIN, 2003) a partir de un modelo elaborado por estudiantes avanzados en la asignatura "Simulación de empresas"; en segundo lugar, describiremos de qué modo se llevó a cabo su enseñanza (MOYANO 2007, 2010a, 2018, 2017); en tercer lugar, se ofrecerán los resultados atendiendo a las entregas que los estudiantes de cada asignatura realizaron a lo largo de la cursada; y por último se discutirán esos resultados en el marco de una propuesta de enseñanza a lo largo del curriculum. Para el análisis de esta experiencia tomaremos también información etnográfica recogida a partir de una entrevista realizada a David Aguirre, docente a cargo de las dos asignaturas y director de la carrera de Licenciatura en Administración. 


\section{Marco teórico}

La lingüística sistémico-funcional ofrece un enfoque que permite analizar los discursos científicos y sociales en el contexto de la cultura. Para eso, se apoya en la noción de género, que define como una combinación de significados que refieren a las variables del campo, el tenor y el modo (MARTIN; EGGINS, 2003; MARTIN; ROSE, 2008). El campo refiere a la actividad social que realiza el discurso, y se realiza a través del significado ideacional; el tenor, a la relación entre los interlocutores de una comunidad, y se realiza mediante el significado interpersonal; y el modo, a la forma que asume el discurso, realizándose mediante el significado textual (MARTIN; ROSE, 2008, 2007). A su vez, cada uno de esos significados se realiza a través del lenguaje en los estratos semántico-discursivo y léxico-gramatical.

Sin embargo, en el discurso científico es común encontrar macrogéneros, esto es, un texto que está formado por varios géneros diferentes (EGGINS; MARTIN, 2003; MARTIN, 1994). Así, los macrogéneros cumplen un propósito social que excede al de cada género que lo integra, como es el caso de los Escenarios.

Por lo tanto, esta perspectiva permite analizar el lenguaje en su contexto y observar de qué manera los hablantes, que se encuentran orientados por un propósito social, seleccionan una serie de opciones del lenguaje que el contexto habilita. Esa selección incluye un lenguaje especializado que contribuye a la realización de dichos propósitos en el discurso de la ciencia y demuestra que en el macrogénero Escenarios se realiza mediante abstracciones, metáforas gramaticales y nominalizaciones (HALLIDAY; MARTIN, 1993; MARTIN; VEEL, 1998; MOYANO, 2013).

En este apartado, entonces, revisaremos los géneros científicos, enfatizando en los Informes. Luego, caracterizaremos el macrogénero Escenarios, atendiendo a su estructura esquemática y los recursos lingüísticos más relevantes para la producción de conocimiento. Entre estos últimos, enfatizaremos en el sistema de IDEACIÓN, que ofrece un modo de analizar cómo se construye la experiencia a través del discurso (MARTIN; ROSE, 2007) y refiere al significado ideacional. Un modo de analizar ese significado es atendiendo a las relaciones taxonómicas, que permiten observar de qué modo se relacionan los ítems léxicos a lo largo del discurso, y que pueden ser de distintos tipos: repetición, sinonimia, contraste, clase y parte (MARTIN; ROSE, 2007). Además, su análisis permite observar la utilización de un lenguaje especializado a través del cual se construyen las entidades en el mundo y, por lo 
tanto, a identificar el género en cuestión, los informes composicionales, que se tratan de géneros centrados en entidades y sus partes (MARTIN; ROSE, 2008).

Luego, para analizar el significado textual, nos centraremos en el sistema de PERIODICIDAD, que permite observar de qué modo se desarrolla la información a lo largo del texto y, para eso, tomaremos el concepto de hiperTema, oraciones tópicas que anticipan la información (MARTIN; ROSE, 2007). Asimismo, ese significado será analizado observando la realización del método de desarrollo (FRIES, 1981) mediante el conjunto de Temas de una fase del texto, considerando los aportes que desde esta perspectiva se han realizado en español. La perspectiva que se asume es la de Moyano (2016), que propone la realización del Tema no marcado en español a través de la concordancia. Por otro lado, se atiende a la realización de Temas marcados mediante Circunstancias. El Tema no marcado es el que permite orientar la mirada hacia un ángulo determinado del campo (MARTIN, 1992; MARTIN; ROSE, 2007), mientras que el Tema marcado colabora con el método de desarrollo, en tanto anticipa una nueva etapa del discurso (MARTIN; ROSE, 2007).

Por último, analizaremos el sistema de VALORACIÓN para observar de qué modo se realizan los significados interpersonales, esto es, qué actitudes son negociadas en el texto y cómo se negocian diferentes posiciones ideológicas en el discurso. Para eso, focalizaremos específicamente en el subsistema de COMPROMISO (MARTIN; WHITE, 2005), que permite observar cómo se realiza la heteroglosia.

\section{Los géneros científicos: el informe composicional}

Según Martin y Rose (2008), la ciencia tiene unas formas específicas de semiotizar el mundo que se organizan en torno a cuatro géneros elementales: los informes, que clasifican y describen; las explicaciones, que ofrecen causas y efectos; los instructivos de observación y experimentación; y los informes de procedimiento, que reportan observaciones y experiencias. De los cuatro, el único que no establece una relación temporal es el informe, ya que su función está ligada a focalizar en una entidad y clasificarla. Esa clasificación puede responder a distintos propósitos, como se observa en la Figura 1, por lo que se reconocen tres variantes prototípicas. 


\begin{tabular}{|l|l|l|l|}
\hline Género & Tipo & \multicolumn{1}{|c|}{ Propósito } & \multicolumn{1}{c|}{ Etapas } \\
\hline Informe & Descriptivo & $\begin{array}{l}\text { Clasificar un fenómeno y describir } \\
\text { sus rasgos }\end{array}$ & Clasificación ^ Descripción \\
\cline { 2 - 5 } & Taxonómico & $\begin{array}{l}\text { Subclasificar un conjunto de } \\
\text { fenómenos, en función de una clase } \\
\text { general }\end{array}$ & Clasificación Tipo \\
\cline { 2 - 5 } & Composicional & $\begin{array}{l}\text { Describir los componentes de una } \\
\text { entidad }\end{array}$ & Clasificación `Componentes \\
\hline
\end{tabular}

Figura 1: Tipos de informes

Como se observa, entonces, en los tres casos hay una primera etapa del género que clasifica una entidad, y según los propósitos, qué se hace con ella. Focalizando en los informes composicionales, esa clasificación presenta una entidad que será descompuesta, por lo que, en las etapas siguientes, se introducen cada uno de sus componentes. Siguiendo a Martin y Rose (2008), estos géneros suelen introducir esos componentes nombrándolos, y en algunos casos, suelen mencionar sus actividades, funciones y/o localizaciones. De esta manera, en la etapa inicial se presenta la entidad como un todo, y en las etapas siguientes se nombran cada una de sus partes.

\section{El macrogénero Escenarios}

Sin embargo, como se anticipó, en el campo científico es habitual que los géneros elementales se combinen en macrogéneros. Siguiendo a Martin (1994, p. 34, traducción propia), "Los macrogéneros son de hecho proporcionales a las estructuras de los complejos clausulares, del mismo modo en que los géneros elementales son proporcionales a las cláusulas". Para analizar esa relación, entonces, Martin (1994) retoma la clasificación realizada por Halliday (1985) sobre los complejos clausulares: paratáctica o hipotáctica, según la interdependencia, y proyección y expansión, según las relaciones lógico-semánticas. A su vez, clasifica la expansión en elaboración, extensión y ampliación, como se observa en la Figura 2. 


\begin{tabular}{|c|c|c|}
\hline \multicolumn{3}{|c|}{ Expansión } \\
\hline Tipo & Función & Estatus \\
\hline Elaboración & $\begin{array}{l}\text { Uno de los géneros especifica o describe algún aspecto del anterior, aunque } \\
\text { sin agregar información nueva. }\end{array}$ & \multirow{2}{*}{$\begin{array}{l}\text { Mismo estatus } \\
\text { (relación } \\
\text { paratáctica) }\end{array}$} \\
\hline Extensión & $\begin{array}{l}\text { Un género agrega información nueva al primero, ya sea reemplazándola u } \\
\text { ofreciendo una alternativa }\end{array}$ & \\
\hline Ampliación & $\begin{array}{l}\text { Un género expande algún aspecto del anterior, ya sea en relación con la } \\
\text { ubicación temporal, espacial, causal, etc }\end{array}$ & $\begin{array}{l}\text { Distinto estatus } \\
\text { (relación } \\
\text { hipotáctica) }\end{array}$ \\
\hline
\end{tabular}

Figura 2: Tipos de relaciones de expansión

Fuente: MARTIN (1994)

A continuación, analizaremos el macrogénero atendiendo al modo en que se desarrolló la propuesta pedagógica, esto es, observando primero su estructura y, luego, los recursos semántico-discursivos y tipo de lenguaje.

\section{Estructura de los Escenarios}

El macrogénero Escenarios está conformado por cinco informes composicionales que, de acuerdo al campo en el que se insertan, se denominan Escenario Actual, Escenario Positivo, Escenario Negativo, Escenario Semi-positivo y Escenario Semi-negativo. Los últimos cuatro constituyen Escenarios futuros respecto del Escenario Actual, de modo que, en el modelo elaborado por los estudiantes, el Escenario Actual estaba ubicado en el 2018 y los cuatro Escenarios futuros, en el 2028. En términos de David Aguirre, este macrogénero permite de producir "posibles alternativas futuras con el propósito de prever los lineamientos de una gestión organizacional" (Entrevista personal). En otras palabras, cada escenario futuro tiene el propósito de identificar alternativas posibles para las situaciones desarrolladas en el Escenario Actual.

A su vez, cada uno de los cinco Escenarios se descompone en dos etapas, tal como indicamos previamente: la identificación de una entidad (el escenario correspondiente en cada caso) y sus componentes (situación económica, social, política, educativa, deportiva, etc.). Podemos observar que el Escenario Semi-positivo comienza de la siguiente manera: (1) "Luego de algunos años de "tormentas" como anunciara en más de una ocasión el ex presidente de la República Argentina por el partido de "Cambiemos" Mauricio Macri (20152019), pareciera que el país comienza a tener un poco de calma." (Modelo de estudiantes). 
Esta oración, que funciona como macroTema, presenta la entidad a descomponer ("el país") y en los párrafos siguientes se introducen sus distintos componentes:

Las políticas iniciadas por el expresidente Axel Kicillof (2023-2027) y continuadas en líneas generales por el actual mandatario Martín Lousteau junto a su vice, Marcelo Tinelli, tanto en materia económica y política como social, están permitiendo equilibrar los números del país. [...]

Otra de las consecuencias, resultado de las políticas aplicadas en los últimos años, tiene que ver directamente con lo social. La desocupación que había alcanzado un alarmante $13 \%$ en 2019 , ha disminuido casi a la mitad desde aquél entonces: la última medición del INDEC indica que la tasa de desocupación de Argentina del segundo semestre de 2028 se ubica en un 6,8\%. La pobreza, que trepaba a un $36 \%$ en 2020, mide actualmente según la UCA (Universidad Católica Argentina) un $24.5 \%$. [...]

Continuando con la educación, este sector junto al de salud fueron de los más beneficiados en la distribución de presupuesto del año actual. [...] (Modelo de estudiantes, resaltado nuestro).

Como se observa, desde una perspectiva ideacional, "las políticas [...] tanto en materia económica y política como social", "lo social", "la desocupación", "la pobreza", "la educación" y "salud”, se desarrollan como los componentes o partes de la entidad "el país".

Esa organización, entonces, es compartida en los cinco Escenarios, de modo que en cada uno de ellos la entidad (la "escena" actual o futura) será descompuesta a partir de sus distintas situaciones (económica, política, social, etc.).

Tomado en su conjunto, el macrogénero se estructura de la siguiente manera:

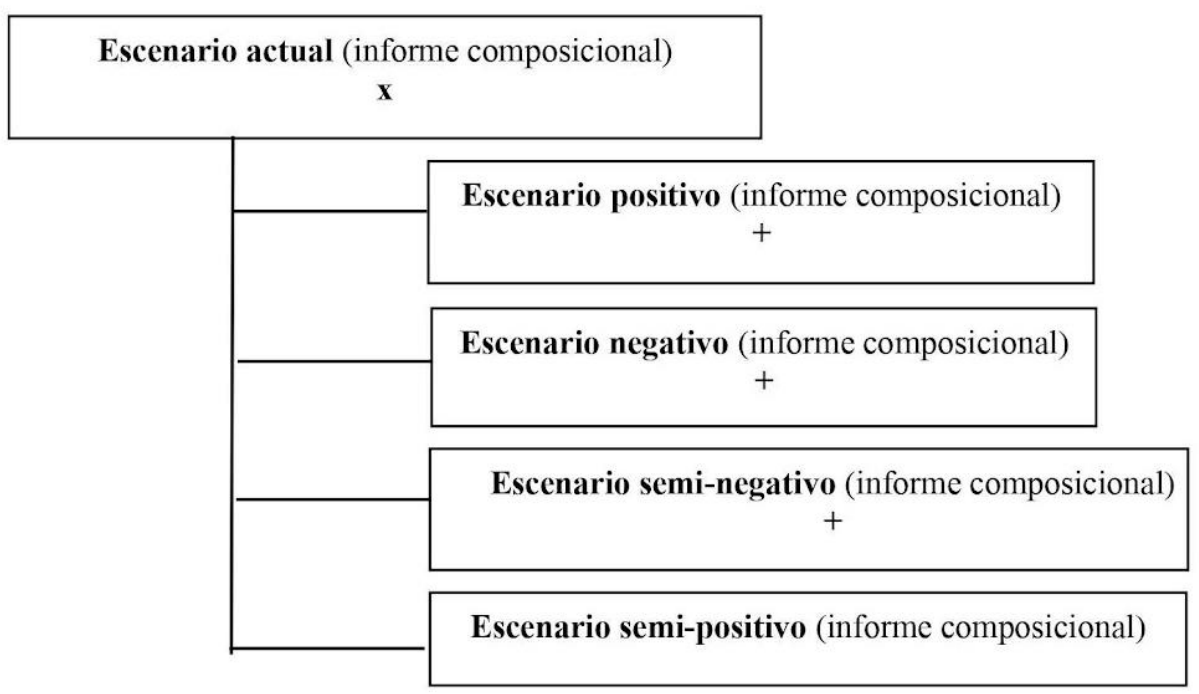

Figura 3: Estructura del macrogénero Escenarios

Fuente: elaborada por las autoras

Como se observa en la Figura 3, en el Escenario Actual se clasifica una escena global, a partir de cada uno de sus componentes (situación económica, social, política, etc.). Luego, 
cada Escenario futuro (Positivo, Negativo, Semi-positivo y Semi-negativo) se ofrece como una alternativa, a diez años de distancia, de ese Escenario Actual, ampliando algunos de sus componentes, según cada caso. De esta manera, los Escenarios futuros funcionan como una co-parte del escenario actual, con el que mantienen una relación de ampliación y, por lo tanto, dependencia. Pero al mismo tiempo, cada escenario futuro es una alternativa de los otros tres, por lo que son independientes entre sí.

En el siguiente fragmento podemos observar la relación de ampliación del Escenario Positivo (3) sobre el Escenario Actual (2):

(2) De acuerdo con el último REM (Relevamiento de Expectativas de Mercado) publicado a fines de Julio por el BCRA, se espera una inflación cercana al 32\% para todo el año 2018, mientras que para algunas consultoras privadas como Eco Go y C\&T Asesores (especializadas en economía y finanzas), la inflación anual tendría un piso de $35 \%$ (Modelo de estudiantes).

(3) Transcurrida una década desde que el país tuviera la inflación más alta del presente milenio, la misma se ubicaría este año en torno al $10 \%$ según el último REM (Relevamiento de Expectativas de Mercado) (Modelo de estudiantes).

Como se desprende de sus nombres, es esperable que en el Escenario Positivo la inflación sea menor a la del Escenario Actual (10\% contra un 32\% a 35\%). Sin embargo, en el Escenario Semi-negativo, esa inflación será del 500\%. De esta manera, la estructura del macrogénero es absolutamente coherente con la práctica social que realiza: funciona como una herramienta de planificación estratégica a largo plazo, que permite anticipar la toma de decisiones ante situaciones imprevistas. En palabras de Aguirre, "el propósito es orientar la gestión organizacional a definir alternativas futuras que contribuyan a reducir la incertidumbre.” (Entrevista personal).

\section{Recursos semántico-discursivos y tipo de lenguaje}

En la experiencia pedagógica que aquí relevamos, luego de presentar la estructura de cada uno de los escenarios y la del macrogénero, se analizan los recursos semánticodiscursivos y el tipo de lenguaje que realizan esas etapas de los géneros. Desde el punto de vista del sistema de IDEACIÓN, entonces, se observan las relaciones taxonómicas posibles: repetición, sinonimia, contraste, clase y parte (MARTIN; ROSE, 2007). En el modelo que realizaron los estudiantes, podemos observar algunas de ellas:

En el período que abarca desde enero 2018 hasta Julio 2018, la inflación acumulada es, según el INDEC, de un 19,6\%. De acuerdo con el último REM (Relevamiento de Expectativas de Mercado) publicado a fines de Julio por el 
BCRA, se espera una inflación cercana al 32\% para todo el año 2018, mientras que para algunas consultoras privadas como Eco Go y C\&T Asesores (especializadas en economía y finanzas), la inflación anual tendría un piso de 35\%. [...]

Continuando en el ámbito económico, otro tema de la actualidad de Argentina es la cotización de la divisa dólar. Finalizando el mes de agosto de 2018, la cotización del dólar para la Venta al público se acerca a casi \$40,00 según el Banco Nación. Recordemos que el día 2 de enero del mismo año, es decir el primer día hábil del mercado financiero, el valor de la divisa para la venta era de $\$ 18.65$ (Modelo de estudiantes, resaltado nuestro).

En ese fragmento, se aborda el componente económico del escenario, y la repetición del ítem léxico "inflación” y "cotización de la divisa dólar" permite desarrollarlo. El uso del sinónimo "el valor de la divisa" atiende a la misma función de ampliar el desarrollo de "la cotización de la divisa dólar". Pero a su vez, "inflación" mantiene una relación de co-parte con "la cotización del dólar" y "el valor de la divisa", en tanto constituyen partes de un todo, el "ámbito económico", y éste último, a su vez, es una co-parte de los otros ámbitos desarrollados en el Escenario Actual. De esta manera, las relaciones taxonómicas realizan el significado ideacional de un modo coherente con el informe composicional, esto es, mantienen una relación de parte/todo o meronimia, tal como se observa en la Figura 4. Asimismo, se observa la utilización de nominalizaciones, lo que permite "construir un mundo de entidades abstractas que se relacionan entre sí.” (MOYANO, 2013, p. 98).

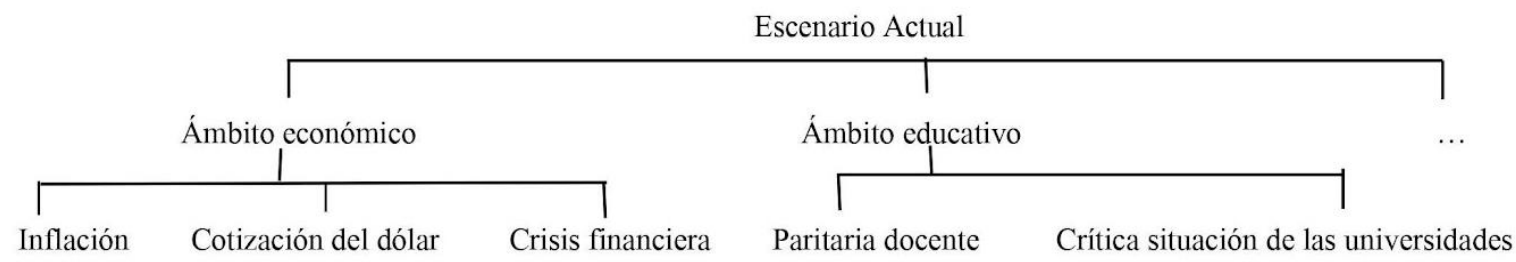

Figura 4: Desarrollo del significado ideacional

Fuente: elaborado por las autoras.

Por otra parte, y como se anticipó, el sistema de PERIODICIDAD, referido al modo, nos permite analizar cómo se realiza el significado textual. Apoyándose en el análisis que Halliday (2002 [1979]) realiza del Tema/Nuevo para el nivel de la cláusula en inglés, Martin (1992) establece que la función del Tema consiste en orientar la mirada del lector hacia un determinado campo, anticipando una cadena léxica perteneciente a un mismo campo semántico. En el Escenario actual, podemos observar esa realización:

(4) En lo que respecta al deporte, por un lado, el retiro de Emanuel "Manu" Ginóbili del mundo profesional del básquet es la gran novedad. A los 41 años de edad y después de 23 años de carrera, Ginóbili es considerado uno de los más 
grandes deportistas argentinos de la historia. El bahiense hizo pública su decisión a través de su cuenta de Twitter (Modelo de estudiantes).

Como se observa en (4), el Tema no marcado "el retiro de Emanuel "Manu" Ginóbili del mundo profesional del básquet" anticipa la cadena léxica que le sigue ("Ginóbili”, "El bahiense"). Pero además, el Tema marcado, realizado por la circunstancia "En lo que respecta al deporte", reorienta la mirada del lector hacia un nuevo componente del escenario, el "aspecto deportivo". Así, también en español, las Circunstancias "contribuyen al método de desarrollo como un modo de organizar el discurso en fases y etapas." (MOYANO, 2016, p. 210, traducción nuestra) y funcionan como "Tema bisagra" (MOYANO, 2010b, p. 16) ya que realizan un cambio en los significados del texto. En los siguientes fragmentos del escenario positivo, observamos una selección similar de los recursos lingüísticos:

En el ámbito judicial, lo más relevante es que ya no quedan detenidos por el caso "los cuadernos", iniciado en 2018. [...]

En lo que respecta al deporte, la gran novedad en el mundo del futbol es la asunción de Diego "el cholo" Simeone como técnico de la Selección Argentina. [...] En cuanto al mundo del básquet, Gabriel Deck, máximo exponente del básquet argentino desde el retiro de Ginóbili volvió a gritar campeón con el Real Madrid, consiguiendo así su cuarto título con la institución española. [...]

En relación con la $\mathbf{T V}$, Susana Giménez confirmó que continuará con su programa el año próximo. [...]

En el contexto internacional, una de las noticias más relevantes es el nuevo acuerdo firmado entre Venezuela y el FMI. [...] (Modelo de estudiantes).

Estos fragmentos sugieren que la selección de las circunstancias en posición inicial se asocia con la función de andamiar una nueva etapa dentro del género. Siguiendo a Moyano, “esta característica del texto en cuestión abona la posibilidad de considerar la existencia de dos capas de Tema para la organización textual en español” (MOYANO, 2010b, p. 16): por un lado, los Temas continuadores que, como en (4), orientan la mirada hacia un determinado ángulo del campo; y por el otro, los Tema bisagra, que "andamian la discontinuidad" (MOYANO, 2010b, p. 16) realizando una nueva etapa del género en cuestión.

Por eso, y desde el nivel del discurso, toda la cláusula (4) funciona como hiperTema (MARTIN; ROSE, 2007), ya que permite anticipar la información que seguirá en los párrafos siguientes, que también referirán al componente del deporte:

En lo que respecta al deporte, por un lado, el retiro de Emanuel "Manu" Ginóbili del mundo profesional del básquet es la gran novedad. A los 41 años de edad y después de 23 años de carrera, Ginóbili es considerado uno de los más 
grandes deportistas argentinos de la historia. El bahiense hizo pública su decisión a través de su cuenta de Twitter.

Por otro lado, Juan Martín del Potro, quien se encuentra tercero en el ranking mundial del tenis, tuvo un debut con brazo firme. Ganó su primera ronda del Us Open contra el estadounidense Donald Young. Hace 12 años que un argentino no se lograba ubicar en el podio del tenis a nivel mundial.

En cuanto al fútbol, la actuación de Argentina en el mundial de Rusia le ha pasado factura a Leo Messi en la carrera por el Balón de Oro. Su rendimiento lo ha dejado sin chances de ganar su sexto trofeo. Tras su desempeño, tomó la decisión de dejar la selección argentina en la que resta del 2018 (Modelo de estudiantes).

Finalmente, el sistema de VALORACIÓN ofrece un modo de analizar el significado interpersonal, lo que permite analizar el tenor del discurso. En términos de COMPROMISO (MARTIN; WHITE, 2005), es común la presencia del discurso referido en los Escenarios. Por lo general, las fuentes citadas ofrecen información estadística, como en (5) y (6), o bien, se introducen las voces de algunos referentes, como en (7) y (8):

(5) Dichos niveles han llegado actualmente a valores inferiores al 5\% anual, según el INDEC, contra valores que alcanzaban entre un $40 \%$ y un $55 \%$ años atrás (Modelo de estudiantes).

(6) La pobreza, que trepaba a un $36 \%$ en 2020 , mide actualmente, según la UCA (Universidad Católica Argentina), un 24.5\% (Modelo de estudiantes).

(7) La diva dijo sentirse en plenitud y afirmó: "Si Mirtha (Legrand) pudo conducir hasta los 94, ¿Por qué yo no?” (Modelo de estudiantes).

(8) El mismo Tinelli afirmó en una conferencia un mes atrás que "la situación del país en las condiciones actuales es insostenible. Argentina necesita reconstruirse y necesita nuevas ideas, y nosotros las tenemos" (Modelo de estudiantes).

En los casos (7) y (8), se realiza una atribución de reconocimiento (MARTIN; WHITE, 2005) a las fuentes citadas mediante el uso de procesos verbales (“dijo", "afirmó”), mientras que en (5) y (6) esa atribución se realiza mediante el uso de "según". En ambos casos, las selecciones dan cuenta de un posicionamiento neutral que "permite al escritor mantener distancia de cualquier relación de acuerdo o desacuerdo" (MARTIN; WHITE, 2005, p. 115) y que el lector entre en contacto con el punto de vista de la fuente citada. Este modo de construir el significado interpersonal se vincula con uno de los propósitos del macrogénero: en términos de Aguirre (Entrevista personal), "estas escrituras modelan las creencias arraigadas en los responsables de documentar el futuro y permiten consensuar un pensamiento futurista", con el objetivo de que "los líderes organizacionales preserven la organización independientemente de lo contextual que se presente en cada escenario.” 


\section{La propuesta pedagógica}

Como anticipamos en la introducción, el trabajo se enmarcó en el Programa de Lectura y Escritura Académica de una universidad argentina, que adapta la propuesta pedagógica llevada a cabo por la Escuela de Sídney en el marco de la lingüística sistémico funcional (MARTIN, 1999; MARTIN; ROSE, 2012). El modelo supone la enseñanza de los géneros académicos y disciplinares mediante un abordaje explícito (BERNSTEIN, 1990) y a través de un andamiaje (WOOD; BRUNER; ROSS, 1976; AUTOR, 2017), reformulado en Rose y Martin (2012), como interacción en un ámbito compartido. Este modelo implica, además, el trabajo conjunto entre el docente disciplinar y el lingüista, quienes, mediante la estrategia de negociación entre pares (MOYANO, 2009; MOYANO; GIUDICE, 2016), establecen una serie de acuerdos para llevar a cabo la enseñanza los géneros académicos.

En concreto, la propuesta se organiza en tres etapas: la Deconstrucción del género, el Diseño del texto y la Edición de las propias producciones (MOYANO, 2007).

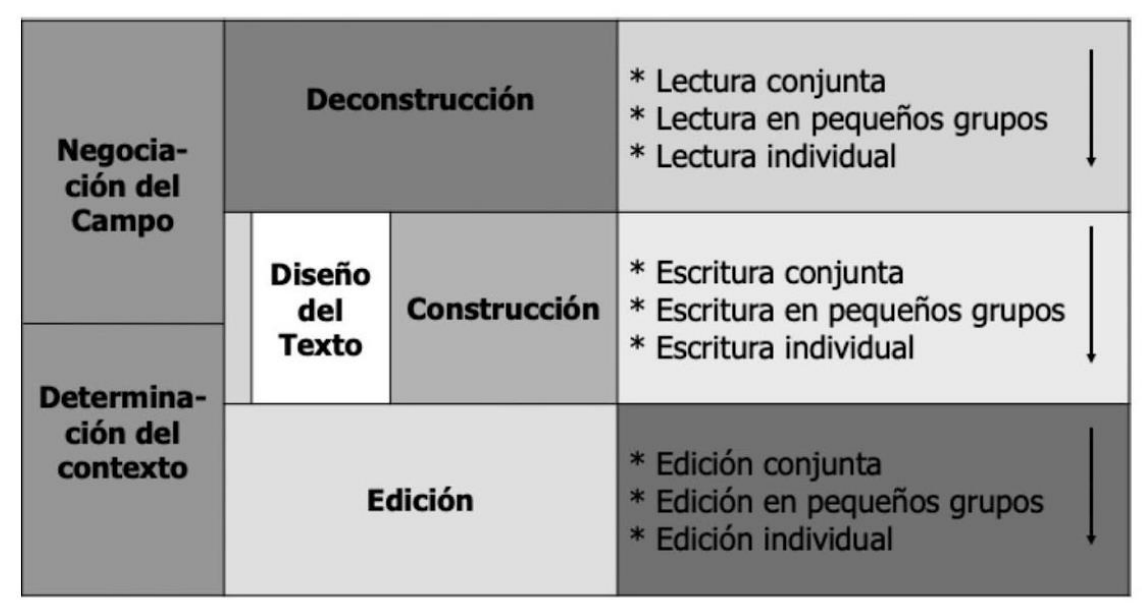

Figura 5: Propuesta didáctica

Fuente: MOYANO (2007)

Como se observa en la Figura 5, entonces, en la primera etapa se analiza el género o macrogénero mediante la lectura conjunta de un modelo, seguida por la lectura en pequeños grupos y luego individual. Esta etapa implica el análisis de la estructura esquemática, y la identificación del tipo de lenguaje y de los distintos recursos semántico-discursivos. Así, la Deconstrucción consiste en una estrategia para el aprendizaje de la escritura, pero también en la enseñanza de una forma específica de lectura (SERPA, 2021). A partir de esa Deconstrucción, los estudiantes elaboran un primer diseño del texto atendiendo a las 
particularidades del género observadas, primero conjuntamente con el docente, luego en pequeños grupos, hasta hacerlo individualmente. Finalmente, se editan esas primeras producciones, a partir de una nueva Deconstrucción, en este caso, con sus propios ejemplares genéricos.

Es interesante destacar en este proceso el pasaje de la heteronomía a la autonomía (MOYANO, 2007, 2017), ya que en la universidad resulta central que los estudiantes adquieran mayor independencia para realizar exitosamente la escritura de géneros académicos y profesionales. Asimismo, la enseñanza explícita y la utilización del metalenguaje necesario contribuyen a incluir a los estudiantes no tradicionales en el habitus académico, entendido como el conjunto de prácticas, lenguajes, estrategias, etc. propios de la universidad (EZCURRA, 2011b).

En el caso de las experiencias que aquí relevamos, la etapa de Deconstrucción consistió en la descripción del macrogénero desarrollada en el apartado anterior. Durante la clase se leyó conjuntamente el mismo modelo para ambos cursos y, para la etapa de Diseño, se les pidió que elaboraran un primer esquema del texto de modo conjunto: qué ámbitos o componentes abordar en cada escenario, cómo organizarlos a lo largo del texto, con qué fuentes trabajar, etc. Luego del Diseño conjunto, cada curso se dividió en grupos y elaboró una primera versión del trabajo que fue entregada. En la etapa de Edición conjunta, la docente retomó la descripción y análisis de la etapa de Deconstrucción para analizar las producciones de los estudiantes, observando aspectos favorables y otros a mejorar. A partir de esa etapa, entonces, cada grupo editó sus producciones para la entrega definitiva.

\section{Recolección de datos}

A continuación se presentará la herramienta para la recolección de datos, atendiendo a cada grupo de estudiantes y dimensiones de análisis del macrogénero. Asimismo, se describirá de qué manera se analizaron esas dimensiones.

Es importante destacar que en ambos grupos todos los estudiantes participaron de las tres etapas del trabajo que se dictaron en modalidad presencial y realizaron la entrega de sus dos versiones. Por último, el trabajo se realizó con la presencia tanto del docente disciplinar como de la profesora del Programa. 


\section{Datos recogidos}

La propuesta pedagógica expuesta más arriba se acompaña de una rúbrica, herramienta que permite vincular la etapa de Deconstrucción con la de Edición y que se construye en base al análisis del macrogénero. De esta manera, se organiza de acuerdo a cuatro ejes: "la estructura esquemática" (la inclusión de los cinco géneros elementales, la organización adecuada en etapas en cada uno de ellos), "los recursos semántico-discursivos" (VALORACIÓN y PERIODICIDAD), "tipo de lenguaje" (nominalizaciones y abstracciones) y "recursos gráficos y normativos" (tildación, ortografía, formato del texto, etc.).

Así, su utilización permite a los y las estudiantes auto-evaluar su desempeño, y evaluar y calificar el proceso de aprendizaje teniendo en cuenta los contenidos que se abordaron en las instancias de Deconstrucción y Edición.

En cuanto a los sujetos, el grupo de la asignatura "Simulación de Empresas" contó con tres estudiantes que elaboraron un trabajo grupalmente, y que presentaron en dos instancias: la primera versión y la versión definitiva. Por su parte, en "Organización de empresas" estuvieron presentes 12 estudiantes que se dividieron en grupos de tres, y presentaron dos versiones del trabajo cada uno.

\section{Análisis de los datos}

Cada dimensión del macrogénero se puntuó con una escala del 1-10. Así, para cada asignatura, se calculó el promedio de las cuatro dimensiones para cada versión del texto. De este modo, el máximo puntaje a obtener es de 10 puntos. Luego, se promediaron esas cuatro dimensiones para obtener una puntuación en cada versión del texto, por asignatura.

\section{Resultados}

A continuación, presentaremos los resultados obtenidos en la elaboración de la primera versión del texto y de su versión definitiva para cada asignatura. Luego, presentaremos los resultados atendiendo a cada una de las dimensiones presentadas en la Deconstrucción y descriptas en la rúbrica. 
Como se observa en la Figura 6, hay una mejora significativa en la edición de las primeras versiones: en Organización de Empresas, las producciones avanzaron un 26\% (sobre 10), mientras que en Simulación de Empresas, avanzaron un 22\%, pero alcanzando, en su versión final, la nota más alta (10). En esta materia, además, la versión inicial tuvo una mejor puntuación que en Organización de Empresas.

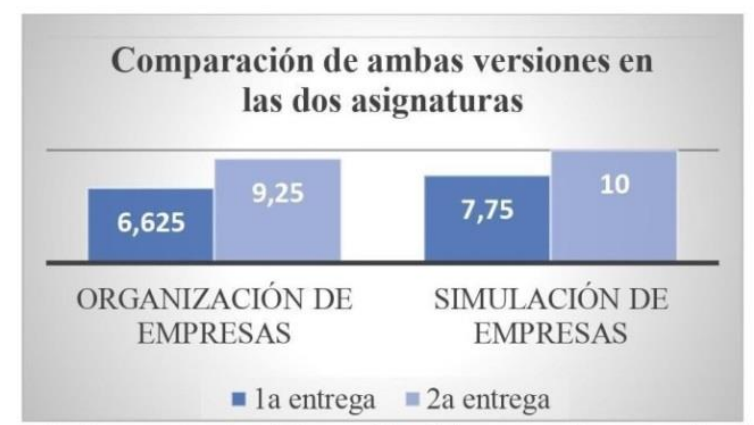

Figura 6: Avance en la elaboración del macrogénero en las dos asignaturas.

Fuente: elaborado por las autoras

En cuanto al rendimiento de los y las estudiantes en relación con las dimensiones de los Escenarios desarrolladas en la rúbrica, podemos observar que en Organización de Empresas (Figura 7), la mayor dificultad en la versión inicial estuvo en los recursos semántico-discursivos (VALORACIÓN y PERIODICIDAD), aunque avanzaron un 32\% para la versión final. En Simulación de Empresas (Figura 8), este eje obtuvo mejor puntuación, y el mayor avance lo presentó en el tipo de lenguaje con un $30 \%$.

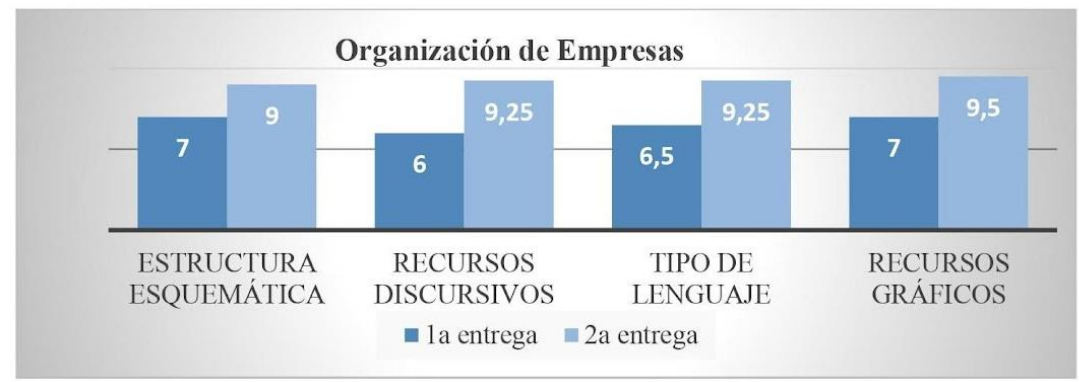

Figura 7: Comparación entre ambas versiones en las dimensiones del macrogénero para Organización de Empresas.

Fuente: elaborado por las autoras 


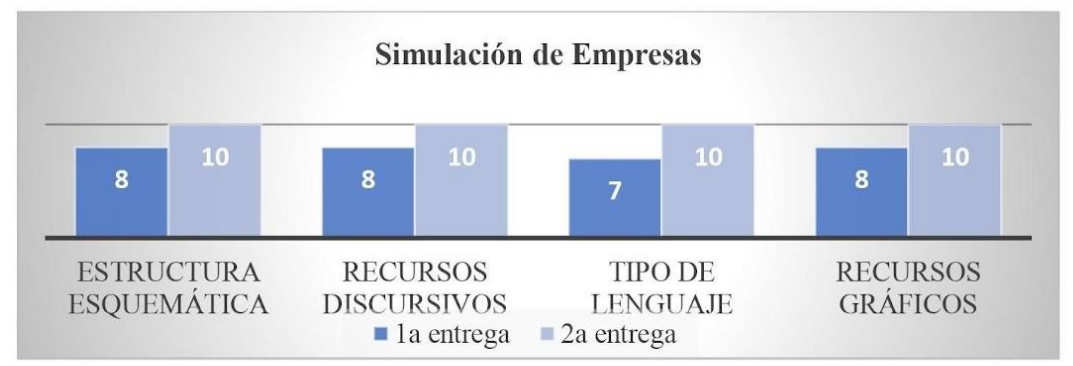

Figura 8: Comparación entre ambas versiones en las dimensiones del macrogénero para Simulación de Empresas.

Fuente: elaborada por las autoras

Desde el punto de vista de los avances, en Organización de Empresas el rendimiento fue mayor entre una versión y otra, pero en Simulación de Empresas se obtuvieron mejores puntuaciones en ambos casos.

\section{Discusión}

Los resultados presentados anteriormente, nos ofrecen varias interpretaciones. En primer lugar, en las dos asignaturas las versiones iniciales, si bien tienen algunos aspectos mejorables, alcanzan una puntuación que, en el ámbito en que se producen, serían aprobadas. Consideramos que en eso influye el trabajo de Deconstrucción, que les ofrece a los y las estudiantes una lectura analítica detenida de un modelo del macrogénero, así como también una guía para su escritura. Asimismo, el trabajo en la etapa del Diseño permite que planifiquen el texto, primero con la docente y luego individualmente. Como ya se mencionó, estas etapas se ofrecen como un andamiaje para la elaboración de un macrogénero, que, sobre todo en las asignaturas de los primeros años, resulta complejo para los estudiantes.

En segundo lugar, la dificultad observada en torno a los recursos semánticodiscursivos en Organización de Empresas, se vincula con que se trata de estudiantes del segundo año de la carrera, por lo que tienden a ejercer una valoración sobre los participantes y los eventos. Por el contrario, en Simulación de Empresas, el avance en esos recursos es significativo comparativamente, por lo que podríamos suponer que ya hay una mayor conciencia genérica en sus estudiantes. Esta hipótesis se sustenta también por dos razones más: por un lado, porque los estudiantes ya se desempeñaban laboralmente en el campo de la Administración; y por el otro, porque ya habían participado en otras asignaturas de años anteriores en las que el PROLEA se implementa, por lo que venían con un mayor entrenamiento en la reflexión sobre el discurso académico. 
Por último, los resultados en ambas asignaturas ponen de relieve la importancia de la etapa de Edición, mediante la que los estudiantes realizan una auto-evaluación de su propia escritura. Acompañada de la rúbrica, esa revisión contribuye a la identificación y apropiación de los recursos lingüísticos de una comunidad disciplinar específica. Nuevamente, la versión inicial del trabajo en Simulación de Empresas evidencia esa apropiación, y enfatiza en la importancia de la enseñanza explícita de la lectura y escritura académica a lo largo del currículum, no sólo para el desempeño en la comunidad académica, sino también, y fundamentalmente, para el desempeño en ámbito profesional.

En definitiva, el análisis del macrogénero Escenarios evidencia el modo en que el lenguaje contribuye a la realización de una práctica profesional y, a la inversa, el modo en que la práctica profesional modela el lenguaje. Por lo tanto, su enseñanza interviene en los aprendizajes disciplinares y en los modos de participación en una comunidad disciplinar específica.

\section{REFERENCIAS}

BERNSTEIN, Basil. Class, codes and control.v. 4 - The structuring of Pedagogic Discourse. London: Routledge, 1990.

CHRISTIE, Frances; MARTIN, James R. (Org.). Genre and institutions. Social processes in the workplace and school. London: Contiuum, 1997.

EGGINS, Suzanne. An introduction to systemic functional linguistics. London: Continuum, 2004.

EGGINS, Suzanne; MARTIN, James R. El contexto como género: una perspectiva lingüístico-funcional. Revista Signos, Valparaíso, v. 36, n. 54, p. 185-205, 2003.

EZCURRA, Ana María. Igualdad en educación superior. Un desafío mundial. Buenos Aires: Universidad Nacional de General Sarmiento, 2011a.

EZCURRA, Ana María. Abandono estudiantil en educación superior. Hipótesis y conceptos. In: GLUZ, Nora (Org.), Admisión a la universidad y selectividad social. Cuando la democratización es más que un problema de "ingresos". Buenos Aires: Universidad Nacional de General Sarmiento, 2011b. p. 23-62.

FRIES, Peter. H. On the status of Theme in English: Arguments from discourse. In: PETÖFI, János; SÖZER, Emel (Org.). Micro and Macro Connexity of Texts. Papers in Textlinguistics. v. 45. Hamburg: Helmut Buske Verlag, 1983. p. 116-152.

HALLIDAY, Michael A. K. An Introduction to Functional Grammar. London: Arnold, 1985. 
HALLIDAY, Michael A.K. Modes of meaning and modes of expression: types of grammatical structure and their determination by different semantic functions. In: WEBSTER, J. (Org.) On Grammar. Collected Works of M.A.K. Halliday. v. 1. London: Continuum, 2002 [1979]. p. 196-218.

HALLIDAY, Michael A.K.; MARTIN, James R. Writing science: Literacy and discursive power. Pittsburgh: University of Pittsburgh Press, 1993.

MARTIN, James R. Theme, method of development and existentiality: The price of reply. Occasional Papers in Systemic Linguistics, Nottingham, v. 6, p. 147-183, 1992.

MARTIN, James. R. Macro-genres: The ecology of the page. Network, Sydney, v. 21, n. 1, p. 29-52, 1994.

MARTIN, James R.; WHITE, Peter. The language of evaluation. Appraisal in English. London: Palgrave, 2005.

MARTIN James R.; ROSE, David. Working with discourse. Meaning beyond the clause. London: Bloomsbury, 2007.

MARTIN, James R.; ROSE, David. Genre relations. Mapping culture. London: Equinox, 2008.

MARTIN, James R.; VEEL, Robert (Org.). Reading Science. Critical and Functional Perspectives on Discourses of Science. London: Routlege, 1998.

MOYANO, Estela I. La escritura académica: una tarea interdisciplinaria a lo largo de la curricula universitaria. Revista Texturas, Santa Fe, v. 4, n. 4, p. 109-120, 2004.

MOYANO, Estela I. Enseñanza de habilidades discursivas en español en contexto preuniversitario: una aproximación desde la LSF. Revista Signos, Valparaíso, v. 40, n. 65, p. 573608, 2007.

MOYANO, Estela I. Negotiating genre: Lecturer's awareness in genre across the curriculum project at the university level. In: BAZERMAN, Charles; BONINI, Adair; FIGUEIREDO, Débora (Orgs.), Genre in a changing world. Perspectives on writing. Colorado: The WAC Clearinghouse and Parlor Press, 2009. p. 449-464.

MOYANO, Estela I. Escritura académica a lo largo de la carrera: un programa institucional. Revista Signos, Valparaíso, v. 43, n. 74, p. 465-488, 2010a.

MOYANO, Estela I. El sistema de tema en español: una mirada discursiva sobre una cuestión controvertida. In: GHIO, Elsa; FERNÁNDEZ, María (Org.). El discurso en español y portugués: Estudios desde una perspectiva sistémico-funcional. Santa Fe: Universidad Nacional del Litoral, 2010b. p. 39-87.

MOYANO, Estela I. El lenguaje de las disciplinas y los géneros de su recontextualización escolar Una aproximación desde la lingüística sistémico-funcional. In: MOYANO, Estela I. (Org.) Aprender ciencias y humanidades: una cuestión de lectura y escritura: aportes para la 
construcción de un programa de inclusión social a través de la educación lingüística. Buenos Aires: Universidad Nacional de General Sarmiento, 2013. p. 31-78.

MOYANO, Estela I. Theme in English and Spanish. Different means of realization for the same textual function. English Text Construction, Amsterdam, v. 9, n. 1, p. 190-220, 2016.

MOYANO, Estela I. Diseño e implementación de programas de lectura y escritura en el nivel universitario: principios y estrategias. Revista Lenguas Modernas, Santiago de Chile, v. 50, p. 47-72, 2017.

MOYANO, Estela I. La enseñanza de la lectura y la escritura académicas mediante un programa a lo largo del curriculum universitario: opción teórica, didáctica y de gestión. Delta, San Pablo, v. 34, n. 1, p. 235-267, 2018.

MOYANO, Estela I.; GIUDICE, Jaqueline. Negotiation between professional peers: Critical strategy for a reading and writing program at the university level. Ilha do Desterro, Florianópolis, v. 69, n. 3, p. 157-17, 2016.

NAVARRO, Federico. El movimiento Escribir a través del Currículum y la investigación y la enseñanza de la escritura en Latinoamérica. In: BAZERMAN, Charles et al. Escribir a través del curriculum. Una guía de referencia. Córdoba: Universidad Nacional de Córdoba, 2008. p. $38-48$.

ROSE, David; MARTIN, James R. Learning to Write, Reading to Learn. Genre, Knowledge and Pedagogy in the Sydney School. London: Equinox, 2012.

SERPA, Cecilia. La enseñanza de la escritura en la educación superior: una experiencia didáctica en torno al macrogénero "Descripción de Flujograma". Íkala, Revista de Lenguaje y Cultura, Medellín, v. 26, n. 1, p. 77-96, 2021.

WOOD, David; BRUNER, Jerome; ROSS, Gail. The role of tutoring in problem solving. Journal of Child Psychology and Psychiatry, London, v. 17, n. 2, p. 89-100, 1976.

Artigo submetido em: 25 abr. 2021

Aceito para publicação em: 09 jun. 2021

DOI: http://dx.doi.org/10.22456/2238-8915.113367 